\title{
Prolonged Abuse of Vasograin Tablets
}

\author{
${ }^{1}$ Akshit Mahajan, ${ }^{2}$ Manish Bathla, ${ }^{3}$ Shazia Anjum, ${ }^{4}$ Saminder Panchal
}

\begin{abstract}
Migraine is a primary headache disorder characterized by moderate to severe recurrent headaches. We describe the case of a 33-year-old male who had been using vasograin tablets in dose of 2 to 3 tablets per day for a period of 16 years without any regular medical advice. In the absence of dose, he experienced various physical and psychological withdrawal symptoms, which were similar to migraine. Patient was managed medically with substitution and supportive therapy. Clinical importance of such cases in medical practice is discussed.
\end{abstract}

Keywords: Chronic headache, Migraine, Vasograin.

How to cite this article: Mahajan A, Bathla M, Anjum S, Panchal S. Prolonged Abuse of Vasograin Tablets. Ind J Priv Psychiatry 2018;12(1):27-28.

Source of support: Nil

Conflict of interest: None

\section{INTRODUCTION}

Migraine is a primary headache disorder characterized by recurrent headaches. Typically the headache appears in one half of the head, is pulsating in nature, and lasts for 2 to 72 hours. Analgesics, triptans, and ergotamines are the most commonly used drugs for the treatment of migraine.

Migraine patients frequently overuse analgesic medications. Frequent use of various type of migraine medication may paradoxically cause an increased frequency of headache attacks. Patients enter into self-perpetuating cycle of daily headaches called chronic headache and use of symptomatic medication. Chronic headache is a common clinical problem. Mathew et $\mathrm{al}^{1}{ }^{1}$ introduced the concept of transformation of episode migraine into chronic daily headache. The term "chronic migraine" has been introduced by and defined by the International Headache Society (IHS).

Chronic daily headache is the commonest type of headache encountered in psychiatric and neuro-outpatients. Majority of these patients are of transformed migraine (TM).

\footnotetext{
${ }^{1}$ Postgraduate Student, ${ }^{2}$ Professor and Head, ${ }^{3}$ Associate Professor, ${ }^{4}$ Senior Resident

${ }^{1-4}$ Department of Psychiatry, MM Institute of Medical Sciences and Research, Ambala, Haryana, India

Corresponding Author: Akshit Mahajan, Postgraduate Student, Department of Psychiatry, MM Institute of Medical Sciences and Research, Ambala, Haryana, India, e-mail: drakshitmahajan1234@gmail.com
}

About $50 \%$ are daily headache and 50\% are transformed from episodic migraine. Transformed migraine is a condition that initially begins as episodic migraine attacks, which then increases in frequency over a period of months to year. Transformed migraine occurs daily or almost daily and appears to be a mixture of tension-type headache and migraine headache.

Vasograin is commonly used for the treatment of vascular headache and migraine. It contains ergotamine tartrate $1 \mathrm{mg}$, caffeine $100 \mathrm{mg}$, paracetamol $250 \mathrm{mg}$, and prochlorperazine maleate $2.5 \mathrm{mg}$. Ergotamine acts as a vasoconstrictor. It also particularly acts peripherally and can damage the epithelium. It can reduce blood supply to the heart. If used beyond a certain amount a day, it can result in lots of life-threatening complications like thrombosis and gangrene. Prochlorperazine is an antipsychotic drug, used as an antiemetic drug. Its prolonged use can cause extrapyramidal symptoms, stiffness, twitching, dystonia, and akathisia. It can also cause tardive dyskinesia and neuroleptic malignant syndrome. Caffeine acts against adenosine in the body and decreases pain. Caffeine triggers the central nervous system and muscle system and improves the metabolism. Paracetamol is an analgesic. Vasograin tablet is one of the frequently overused medicines in transformed headache. Because of its side-effect profile and availability of better alternative medicines, vasograin is not recommended for its regular use in patients with migraine.

\section{CASE REPORT}

We describe a 33-year-old married male patient with migraine who was regularly self-medicating 1 to 3 tablets of vasograin for more than 16 years. The patient presented with complaints of headache, vomiting, giddiness, and decreased sleep. Headache was one sided, severe in intensity, and episodic in nature lasting 3 to 4 hours a day; 16 years back this patient was started on vasograin sos by a family doctor. Following that, for regularly 16 years he had been taking 1 to 3 tablets vasograin daily without any subsequent consultation. In the absence of vasograin, he complained of headache, nausea, and vomiting and again started using vasograin. Then he did not miss vasograin even for a single day in the past 16 years. All his investigations including computed tomography scan were normal. He was advised to stop the tablet vasograin and managed with tablet Flupirtine SR $400 \mathrm{mg}$ OD and tablet 
Pregabalin $75 \mathrm{mg}$ TDS. Both of these medicines were gradually reduced and finally stopped within a period of 6 months. At the time of last visit he was maintaining well without migraine headache.

\section{DISCUSSION}

In this case, there are two possibilities: First is chronic daily headache changed into TM, second is medication overuse headache $(\mathrm{MOH}) .^{2}$ In the clinical setting it is quite difficult to distinguish these two entities. There is a need to remain aware and sensitive about these clinical problems in medical practices. If such cases remain undiagnosed or untreated, there are chances for many drug-induced medical complications.

Medication overuse headache is a worldwide health problem with a prevalence of 1 to $2 \% .^{3,4}$ It is a severe form of headache where the patients often have a long history of headache and unsuccessful treatments. Medication overuse headache is characterized as chronic headache and overuse of different headache medications. There is a clear consensus regarding optimal strategy for the management of $\mathrm{MOH}$.

\section{REFERENCES}

1. Mathew NT, Reuveni U, Perez F. Transformed or evolutive migraine. Headache 1987 Feb;27(2):102-106.

2. Verma A. Transformed migraine: a study of 420 consecutive patients from central India. Ann Neurosci 2007 Apr;14(2): 37-40.

3. Diener HC, Limmroth V. Medication-overuse headache: a worldwide problem. Lancet Neurol 2004 Aug;3(8):475-483.

4. Kristoffersen ES, Lundqvist C. Medication-overuse headache: a review. J Pain Res 2014 Jun;7:367-378. 